\title{
Interleukin-33 suppresses Notch ligand expression and prevents goblet cell depletion in dextran sulfate sodium-induced colitis
}

\author{
HIROTSUGU IMAEDA $^{1}$, AKIRA ANDOH ${ }^{2}$, TOMOKI AOMATSU ${ }^{1}$, KAZUHIKO UCHIYAMA $^{3}$, \\ SHIGEKI BAMBA $^{1}$, TOMOYUKI TSUJIKAWA ${ }^{1}$, YUJI NAITO ${ }^{3}$ and YOSHIHIDE FUJIYAMA ${ }^{1}$ \\ Department of Medicine, Graduate School of Medicine, Shiga University of Medical Science, Seta \\ Tsukinowa, Otsu 520-2192; ${ }^{3}$ Division of Molecular Gastroenterology and Hepatology, Kyoto Prefectural \\ University of Medicine, Hirokoji Kawaramachi, Kamigyo-Ku, Kyoto 602-8566, Japan
}

Received April 21, 2011; Accepted May 23, 2011

DOI: $10.3892 /$ ijmm.2011.718

\begin{abstract}
Interleukin (IL)-33 is a cytokine belonging to the IL-1 family. IL-33 plays an important role in Th2 immune responses, and induces goblet cell hyperplasia in the intestinal mucosa. In this study, to elucidate the molecular mechanisms underlying IL-33-induced goblet cell hyperplasia, we investigated how IL-33 modulates the Notch signaling pathway in dextran sulfate sodium (DSS)-induced experimental colitis. DSS colitis was induced in BALB/c mice with intraperitoneal administrations of IL-33 ( $1 \mu \mathrm{g} /$ body) every $48 \mathrm{~h}$. Tissue samples were evaluated by standard immunohistochemical procedures. The mucosal mRNA expression of the Notch ligands was analyzed by a real-time polymerase chain reaction. The mucosal mRNA expression of Notch ligands [Jagged1 (Jag1) and Delta-like (Dll) 1 and 4] was significantly increased in DSS-colitis mice. IL-33-induced goblet cell hyperplasia in the control mice. In the DSS-colitis mice, the goblet cells were depleted in the colon, but IL-33 completely prevented goblet cell depletion in the DSS-colitis mice. IL-33 induced a significant decrease in Jag1 and D114 mRNA expression in the mucosa of the control mice. Mucosal mRNA expression for Jag1, Dll1 and 4 was significantly elevated in the DSS-colitis mice, but this elevation was significantly blocked by the administration of IL-33. IL-33 dose-dependently decreased Jag1 mRNA expression in mouse colonic subepithelial myofibroblasts. In contrast to its preventive effects on goblet cell depletion, IL-33 aggravated DSS colitis. IL-33 prevented goblet cell depletion via its inhibitory actions against Notch ligand expression in DSS colitis, but exacerbated the disease activity. IL-33 plays two counter actions in mucosal inflammation; the first is a protective action via goblet cell induction, and the second is a pro-inflammatory action as a Th2 cytokine.
\end{abstract}

Correspondence to: Dr Akira Andoh, Division of Mucosal Immunology, Graduate School of Medicine, Shiga University of Medical Science, Seta Tsukinowa, Otsu 520-2192, Japan

E-mail: andoh@belle.shiga-med.ac.jp

Key words: interleukin-33, goblet cell, Jagged1, Delta-like 1

\section{Introduction}

Interleukin (IL)-33 is a cytokine belonging to the IL-1 family, which was recently identified as a ligand for the IL-1 receptor (IL-1R) family member ST2 (1). Like IL-1 and IL-18, IL-33 can be proteolytically cleaved in vitro by caspase-1 to generate a mature form of the protein. The injection of IL-33 into mice induces broad proinflammatory effects, inducing eosinophilia, splenomegaly, and increased serum levels of IL-5 and IgE $(1,2)$. Recent studies also suggest a role for IL-33 in the development of tissue fibrosis (3-5). Based on these actions, IL-33 has been considered to play an important role in Th2 immune responses. Interestingly, IL-33 has been reported to induce goblet cell hyperplasia, and stimulates mucous production at mucosal surfaces in vivo $(1,6,7)$.

Goblet cells are mucus secretory intestinal epithelial cells (IECs) $(8,9)$. They secrete several mucins, and form a mucous layer, which is regarded as a barrier between the luminal contents and the epithelial surface. These cells are derived from stem cells located in the basal part of the crypt, and their differentiation is dependent on different molecular pathways and the transcription factors of the Notch and Wnt signaling pathways (10-13).

Notch signaling functions as a regulator of the differentiation and proliferation of IECs, and contributes to the maintenance and homeostasis of the intestinal mucosa $(8,10)$. Interactions between Notch receptors (Notch1-4) and their ligands [Jagged1 (Jag1) and 2, and Delta-like (Dll) 1, 3 and 4] result in the proteolytic cleavage of Notch by the $\gamma$-secretase and other proteases, and consequently the intracellular domain of Notch is released and translocated into the nucleus to regulate the transcription of downstream target genes, such as the hairy and enhancer of the split (Hes) gene family members $(8,10)$. Hes1 is a basic helix-loop-helix (bHLH) transcriptional repressor induced by the Notch signal, whereas the mouse atonal homolog 1 (Math1) is another bHLH transcription factor that is suppressed by Hes1 (14). A study on Hes1 knockout (KO) mice demonsrated that a depletion of Hes1 resulted in significant increases in secretory lineage cells (e.g. goblet, Paneth and enteroendocrine cells) and decreases in absorptive cells (15). Other studies have shown that Math1-mutant mice failed to develop most secretory lineage cells, and were colonized by absorptive cells (14). In mouse intestinal tissue, 
Table I. PCR primers used in this study.

\begin{tabular}{lll}
\hline Gene (NCBI ID) & Orientation & Sequence (5'-3') \\
\hline Mouse Jag1 & Forward & TGTGTCCCGGTGGCTGGGAA \\
$($ NM_013822.5) & Reverse & GGCCACCCGTCCATTCAGGC \\
Mouse Dll1 & Forward & GACCCCGCCTTCAGCAACCC \\
$($ NM_007865.3) & Reverse & GTCCCCGCAGGTGAAGTGGC \\
Mouse Dll4 & Forward & GGGGCAGCATGCCTGGGAAG \\
$($ NM_019454.3) & Reverse & CGGTGCAACTCTTGGCGGGT \\
$\beta$-actin & Forward & GTGGGCCGCCCTAGGCACCA \\
& Reverse & CGGTTGGCCTTAGGGTTCAGGGGGG \\
\hline
\end{tabular}

the pharmacological blockage of Notch signaling by $\gamma$-secretase inhibitors down-regulated Hes1, and induced an increase in the number of goblet cells and enteroendocrine cells $(16,17)$. Thus, the Hes1-mediated repression of Math1 contributes to the regulation of the decision of secretory vs. adsorptive lineage.

In this study, to clarify the molecular mechanisms underlying IL-33-induced goblet cell hyperplasia, we investigated how IL-33 modulates the Notch signaling pathway in dextran sulfate sodium (DSS)-induced experimental colitis in mice.

\section{Materials and methods}

Induction of colitis. Six to eight week-old male BALB/c mice were purchased from Charles River Japan (Kanagawa, Japan). They were acclimated for one week before the experiment, and were housed individually in a room maintained at $22^{\circ} \mathrm{C}$ under a 12-h day/night cycle throughout the experiments. Mouse IL-33 was purchased from R\&D systems (Minneapolis, MN). IL-33 ( $1 \mu \mathrm{g} /$ body) was administered intraperitoneally every $48 \mathrm{~h}$ for the duration of the experiment. This study protocol was approved by the Animal Care and Use Committee of the Shiga University of Medical Science (Otsu, Japan).

Cells. Mouse colonic subepithelial myofibroblasts were prepared according to the method reported by Mahida et al (18). The culture conditions were described in a previous report (19). These studies were performed on passages 3-6 of myofibroblasts isolated from six resection specimens. The YAMC (Young Adult Mouse Colon) cell line was used as the mouse colonic epithelial cell. This cell line is derived from the immortimouse that expresses a temperature-sensitive SV40 large $\mathrm{T}$ antigen transgene under the control of the IFN- $\gamma$-sensitive segment of the class MHCII promoter (20). The cells were a generous gift of Dr R. Whitehead (Vanderbilt University, Nashville, TN). The cells were cultured according to recently described methods (20).

Assessment of inflammation in DSS-induced colitis. A daily clinical assessment of the DSS-induced colitis was performed, including a measurement of food intake and body weight, an evaluation of stool consistency, and the presence of blood in the stools by the guaiac paper test. The stool consistency was assessed using the following four point-scale: 0 , normal;
1, soft; 2, very soft but formed; and 3, liquid. The intensity of the guaiac paper test was scored by the following scale: 0 , negative; 1 , faintly blue; 2 , moderately blue; 3 , dark blue; and 4 , blood visible. A validated clinical disease activity index ranging from 0 to 4 was calculated using the following parameters: stool consistency, presence of fecal blood, and changes in body weight (21). The mice were sacrificed on Day 13 .

Detachment of the epithelial layer. The epithelial layer of the colonic mucosa was detached from the subepithelial layer using the method described by Mahida et al (18).

Histology and immunohistochemistry. A histological examination was performed on three samples from each animal. The samples were washed for 4 days with several changes of $0.1 \mathrm{M}$ PB containing $15 \%$ sucrose, and then embedded with $10 \%$ gelatin, cut into $15 \mu \mathrm{m}$ longitudinal sections, and stained with Alcian blue. All histological evaluations were performed in a blinded fashion using a validated scoring system (22). Antibodies [goat anti-Jag1 antibody (Santa Cruz Biotechnology sc-34473, Santa Cruz, CA) and goat anti-Delta-4 antibody (Santa Cruz Biotechnology)] were used. Biotin-labeled anti-goat IgG (Vector Laboratories, Burlingame, CA) and avidin-biotin peroxidase complex (Vector Laboratories) were used for detection. Color development was performed using 3,3'-diaminobenzidine (DAB) (Sigma-Aldrich, St. Louis, MO).

Real-time polymerase chain reaction (PCR). The mRNA expression of Notch ligands in the samples was assessed by real-time PCR analysis. The oligonucleotide primers used in this study are described in Table I. Real-time PCR was performed using a LightCycler 2.0 system (Roche Applied Science, Tokyo, Japan). The PCR was conducted using a SYBR-Green PCR Master Mix (Applied Biosystems, Foster City, CA). The data were normalized against $\beta$-actin for each gene.

Statistical analysis. Statistical analyses were performed using a one-way ANOVA with Scheffe's-post-hoc test or the KruskalWallis test when appropriate. A two-way ANOVA for repeated measures was used to test for group and time effects on the clinical data (e.g., disease activity index). ${ }^{*} \mathrm{P}$-values $<0.05$ were considered to be statistically significant. 

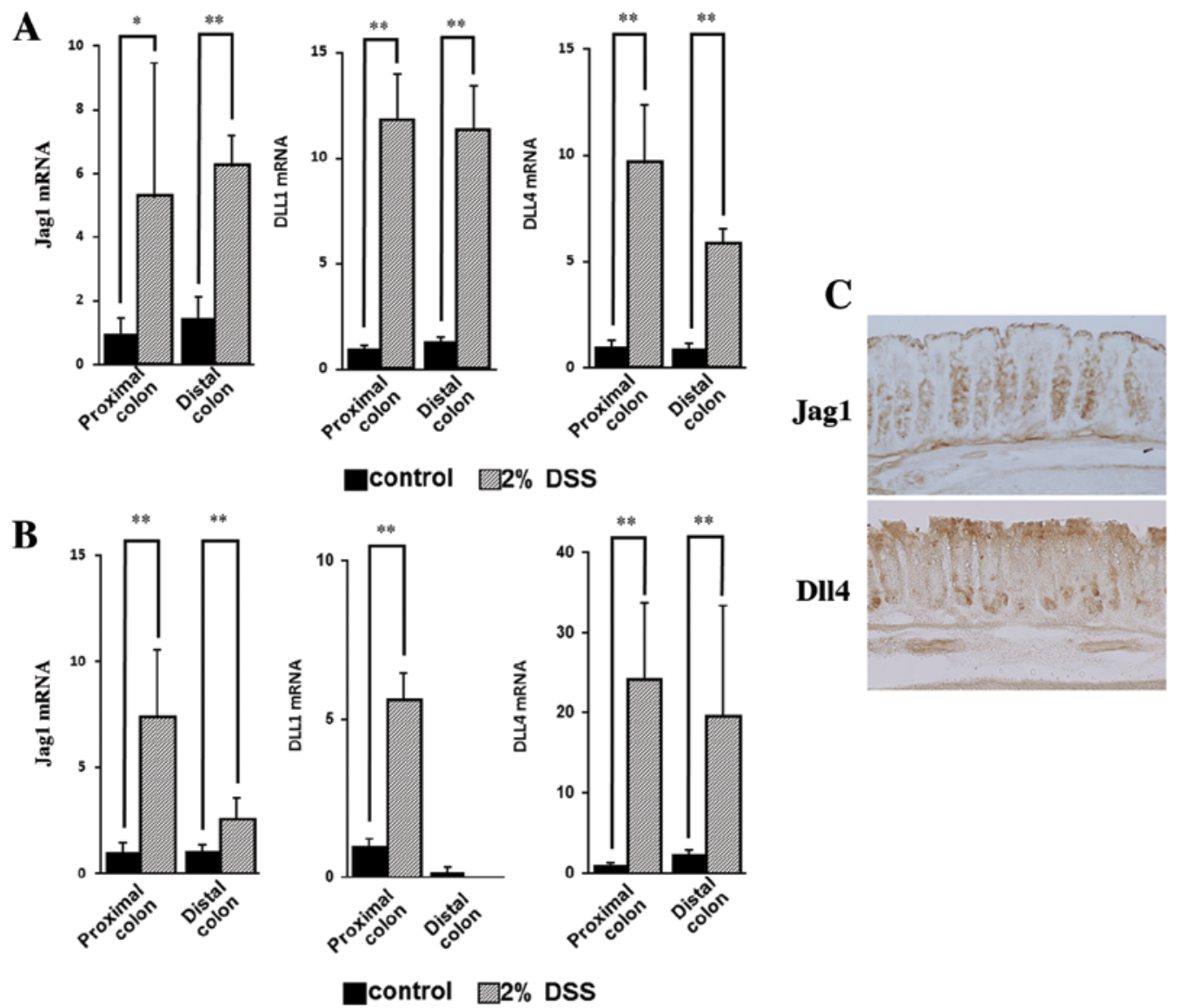

Figure 1. Notch ligand mRNA expression in dextran sulfate sodium (DSS) colitis. (A) The mucosal mRNA expression of the Notch ligands [Jagged1 (Jag1), Delta-like (Dll) 1 and 4] in the proximal and distal colonic mucosa of DSS colitis. The mRNA expression of the Notch ligands was determined by real-time PCR. The data were expressed as the Notch ligand mRNA expression relative to $\beta$-actin mRNA expression (mean \pm SD from 4 different experiments). ${ }^{* *} \mathrm{P}<0.01$; "P<0.05. (B) The mRNA expression of Notch ligands in the epithelial and subepithelial layers. The epithelial layer was detached from the subepithelial layer by enzymatic treatment. The Notch ligand mRNA expression in each layer was determined by real-time PCR. The data were expressed as the Notch ligand mRNA expression relative to the $\beta$-actin mRNA expression (mean \pm SD from 4 different experiments). ${ }^{* *} \mathrm{P}<0.01$. (C) Immunohistochemical staining for Jag1 and D114 in the inflamed mucosa of DSS colitis (magnification, $\mathrm{x} 20$ ).

\section{Results}

Initially, we analyzed the mRNA expression of the Notch ligands (Jag1, Dll1 and 4) in DSS colitis. The mRNA expressions of Jag1, Dll1 and Dll4 were all significantly increased in the mucosa of the proximal and distal colon of DSS-colitis mice (Fig. 1A). This increase was also observed in the epithelial layers containing epithelial cells and subepithelial myofibroblasts detached by enzymatic treatment. The mRNA expression of Jag1 and Dl14 was also significantly elevated in the remaining subepithelial tissues. Representative immunohistochemical staining pictures of Jag1 and Dll4 are shown in Fig. 1C.

Previously, IL-33 has been reported to induce goblet cell hyperplasia in mice. Therefore, we evaluated this phenomenon in DSS-colitis mice. Alcian blue staining showed that IL-33 induced goblet cell hyperplasia in the control mice (Fig. 2A). In the DSS-colitis mice, the goblet cells were depleted in the colon, but in the IL-33-treated DSS-colitis mice, the goblet cells were completely unaffected. To examine the role of Notch signaling, we investigated the mucosal mRNA expression of Notch ligands. IL-33 treatment induced a significant decrease in Jag1 and Dll4 mRNA expression in the mucosa of the control mice (Fig. 2B). IL-33 also exhibited a significant inhibitory effect on the DSS-induced mRNA expression of Notch ligands in DSS-colitis mice.

To investigate the in vitro effects of IL-33 on Notch signaling, we used mouse subepithelial myofibroblasts isolated from normal colonic tissue and the mouse colonic epithelial cell line YAMC (Fig. 3). IL-33 dose-dependently decreased Jag1 mRNA expression in subepithelial myofibroblasts, but had no effect on YAMC cells. IL-33 did not exhibit any inhibitory effects on Jag1 mRNA expression in other human colonic epithelial cell lines, such as HT-29 and Caco-2 cells (data not shown).

Next, we evaluated the effect of IL-33 on DSS colitis. The body weight of DSS-treated mice decreased gradually (Fig. 4a). Contrary to our initial expectations, IL-33 treatment induced a much more severe body weight loss. After Day 11, the body weight was significantly lower in the IL-33-treated DSS-colitis mice than in the control DSS-colitis mice. In contrast, control mice treated with IL-33 showed a gradual increase in body weight, and by Day 7, there was a significant difference in body weight between the IL-33-treated and control mice. The disease activity index (DAI) on Day 13 was significantly higher in IL-33-treated DSS mice than in the control DSS 
A

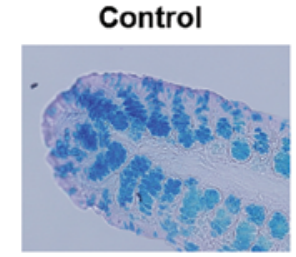

IL-33

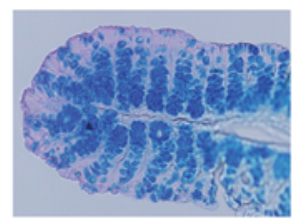

DSS

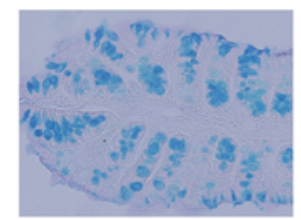

DSS + IL-33

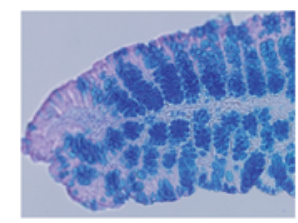

B a

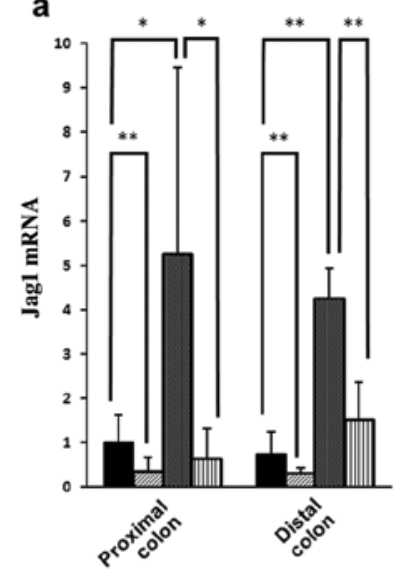

b

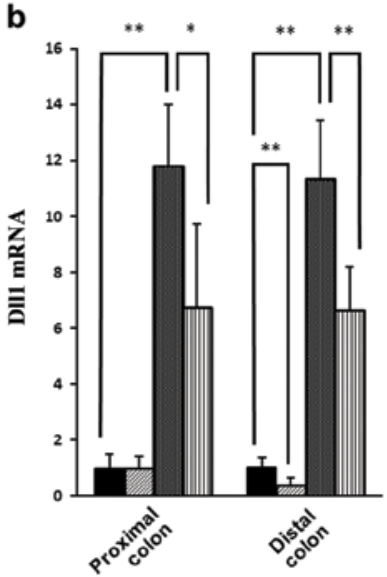

c

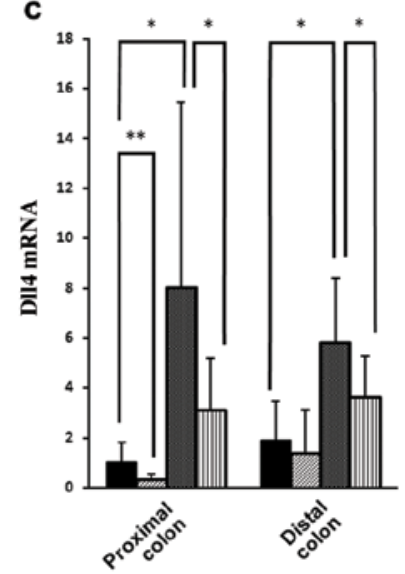

control PBS ip $\square$ control IL-33 ip $\square 2 \%$ DSS PBS ip

Figure 2. Effects of IL-33 on Notch ligand mRNA expression in DSS-colitis mice. (A) Alcian blue staining of the colonic mucosa. (B) Notch ligand mRNA expression in dextran sulfate sodium (DSS) colitis. (a) The mucosal mRNA expression of Notch ligands in the proximal and distal colonic mucosa of DSS- and IL-33-treated DSS-colitis mice. The data were expressed as the Notch ligand mRNA expression relative to $\beta$-actin mRNA expression (mean \pm SD from 4 different experiments). ${ }^{* *} \mathrm{P}<0.01 ;{ }^{*} \mathrm{P}<0.05$.

A

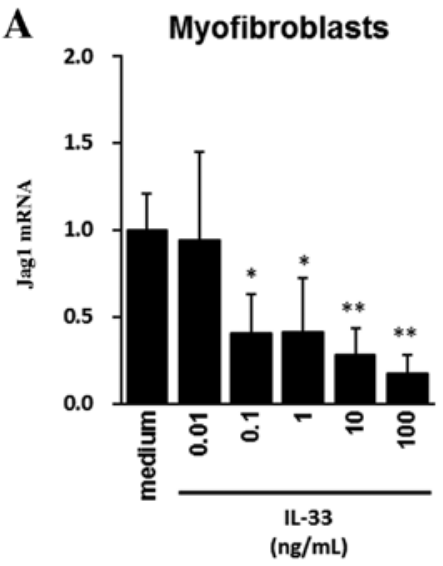

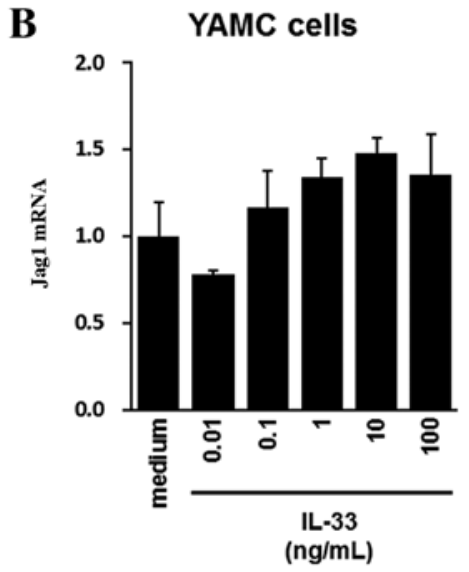

Figure 3. Effects of IL-33 on Jag1 mRNA expression (A) in mouse subepithelial myofibroblasts and (B) the mouse colonic epithelial cell line YAMC. IL-33 dose-dependently decreased the Jag1 mRNA expression in subepithelial myofibroblasts, but had no effect on YAMC cells. The data were normalized vs. $\beta$-actin for Jag1. All values are expressed as means $\pm S D(n=5) . ~ P<0.05,{ }^{* *} \mathrm{P}<0.01$, a significant difference from the values for medium alone.

mice (Fig. 4B). The total colon length was significantly shorter in the IL-33-treated DSS mice than in the control DSS mice (Fig. 4B and C). Thus, IL-33 aggravated DSS colitis in mice.

\section{Discussion}

The receptor for IL-33 is composed of two subunits, IL-1RAcP and ST2 (1). Consistent with the restricted expression of ST2 by Th2-associated cell types (Th2 cells, basophils, eosinophils, invariant NKT cells, and mast cells) $(1,6,7)$, the systemic administration of recombinant IL-33 induces Th2 cytokine production, elevated plasma IgE levels, and eosinophilia (1). In addition, one characteristic effect of systemic IL-33 administration is goblet cell hyperplasia in the lung and gut $(1,6)$. A study by Kondo et al demonstrated that an intraperitoneal injection of IL-33 induced airway goblet cell hyperplasia, even in RAG-2 KO mice which lacked both $\mathrm{T}$ and $\mathrm{B}$ cells (6). In addition, the peritoneal administration of IL-33 into IL-13 KO mice did not induce goblet cell hyperplasia (6). These findings suggest that $\mathrm{T}$ and $\mathrm{B}$ cells are not involved in IL-33-induced 
A

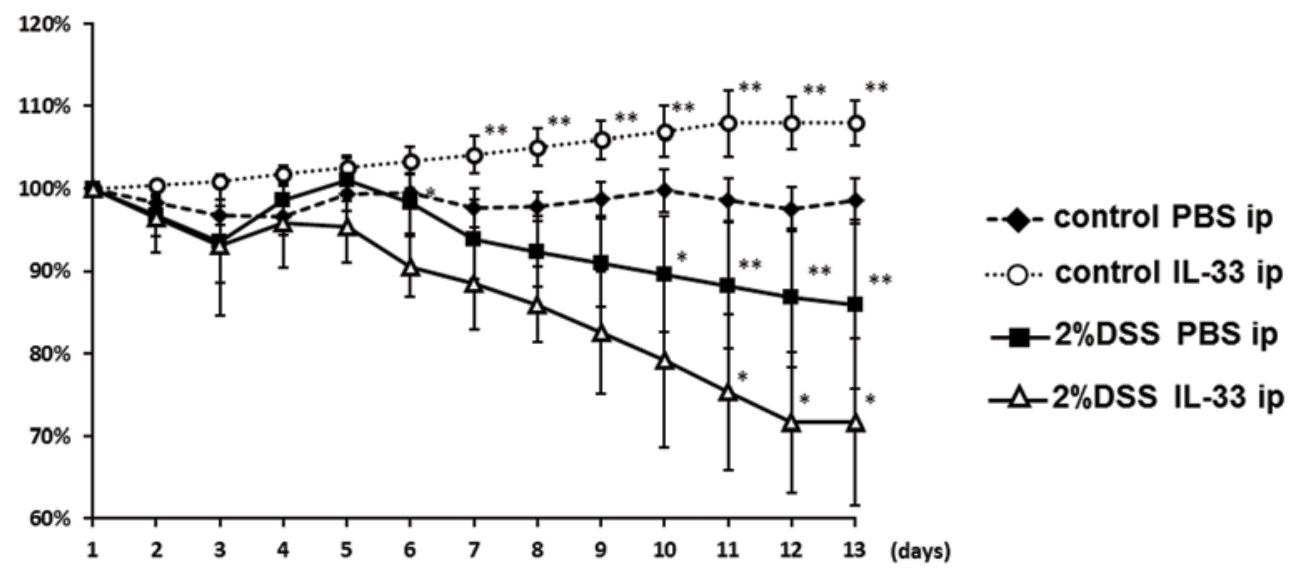

B

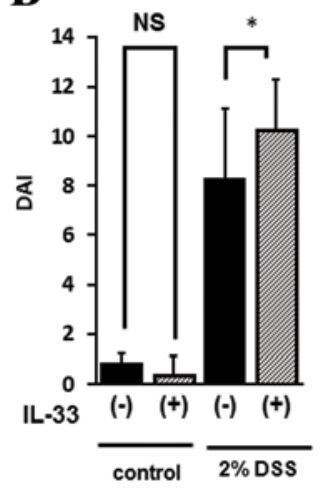

$(\mathrm{mm})$

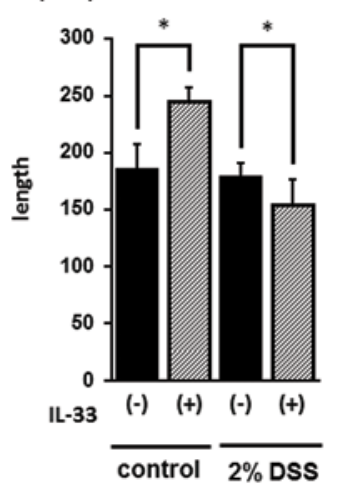

C

Control PBS ip

$2 \%$ DSS PBS ip

$2 \%$ DSS IL-33 ip

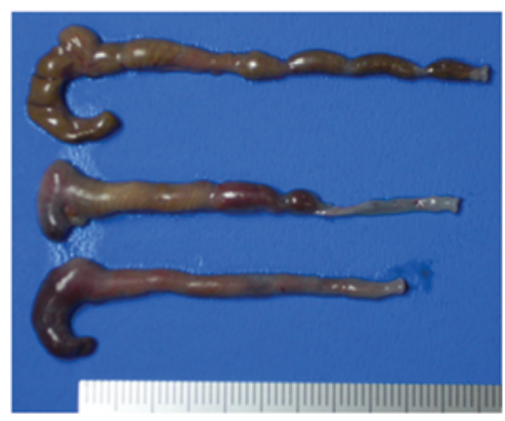

Figure 4. Effects of IL-33 on DSS colitis in mice. (A) Changes in body weight. The weight of each individual mouse was followed daily. The data represent means $\pm \mathrm{SD}$ ( $\mathrm{n}=5$ mice/group). ${ }^{* *} \mathrm{P}<0.01,{ }^{*} \mathrm{P}<0.05$, the evaluation was performed between the DSS and IL-33/DSS groups, DSS and control groups, and IL-33 and DSS groups. (B) Effects of IL-33 on the disease activity index (DAI) and colonic length. The scoring criteria were described in the Materials and methods. The data represent means $\pm \mathrm{SD}$ ( $\mathrm{n}=5$ mice/group). ${ }^{*} \mathrm{P}<0.05$. (C) Representative photograph of the colon isolated from a DSS-colitis mouse.

goblet cell hyperplasia, and support a critical role for IL-13. However, the contribution of the Notch signaling pathway to IL-33-mediated goblet cell hyperplasia remains unclear.

The current study demonstrated for the first time that the mRNA expression of Notch ligands (Jag1, Dll1 and Dll4) was enhanced in the inflamed mucosa of DSS colitis, and was accompanied by goblet cell depletion. The molecular events underlying goblet cell depletion in DSS colitis have been reported by Okamoto et al (16). In this study the goblet cell depletion in DSS colitis was suggested to occur through Hes1 activation, a downstream signaling molecule of the Notch receptor, but the investigators did not analyze the changes in Notch ligand expression. Combined with our observations in this study, the pathophysiology of goblet cell depletion in DSS colitis can be explained by the hypothesis that the enhanced expression of Notch ligands following Notch signaling activation (Hes1 activation) repressed the Math1 activation, and directed the IEC fates toward an absorptive lineage.

There are no reports describing the effects of IL-33 on goblet cell depletion in DSS colitis. We showed that an intraperitoneal injection of IL-33 prevented goblet cell depletion in DSS colitis. This was accompanied by significant inhibitory effects of IL-33 on the elevated mucosal mRNA expression of Notch ligands. Furthermore, IL-33 dose-dependently inhibited Jag1 mRNA expression in mouse subepithelial myofibroblasts. Although the precise molecular mechanisms underlying the inhibitory effects of IL-33 on Notch ligand expression remain elusive, it became clear that IL-33 not only induces goblet cell hyperplasia in the normal colon, but also prevents goblet cell depletion under pathological conditions via a modulation of Notch ligand expression. Since goblet cell hyperplasia induced by IL-33 could not be observed in IL-13 KO mice (6), the contribution of IL-13 to IL-33-mediated events in DSS colitis should be investigated in the future.

Goblet cells secrete several mucins and form a mucous layer, which functions as a barrier between the luminal contents and the epithelial surface $(8,9)$. Based on this concept, we initially expected that IL-33 prevents the development of DSS colitis via a blockade of goblet cell depletion. However, IL-33induced an exacerbation of the disease activity of DSS colitis. Similar observations have been reported by Okamoto et al (16). They reported that an inhibition of Notch signaling by a $\gamma$-secretase inhibitor at the recovery stage of DSS colitis exacerbated the severity of the mucosal damage and inflammation. They also showed that Notch activation is required not only to differentiate the IEC fates towards absorptive cells, but also to proliferate IEC precursor cells, including stem cells (16). This means that IECs need Notch activation to undergo proliferation and development from precursor cells at the recovery stage of mucosal inflammation. In contrast, Shinoda et al (8) recently reported that the early-stage blockade of Notch signaling by a $\gamma$-secretase inhibitor inhibits the depletion of 
goblet cells, and ameliorates DSS colitis. They administered a $\gamma$-secretase inhibitor to block Notch signaling upon the start of DSS exposure. They suggested that maintaining goblet cells by Notch signal inhibition contributed to an amelioration of DSS colitis. In this study, early treatment with IL-33 prevented goblet cell depletion, but also exacerbated the colitis. It is likely that the pro-inflammatory nature of IL-33, such as cytokine and chemokine induction $(1,4)$, might have contributed to the exacerbation of colitis despite the maintained barrier function of goblet cells.

In conclusion, IL-33 prevented goblet cell depletion in DSS colitis via its inhibitory actions on the expression of Notch ligands, but exacerbated the disease activity. These observations suggest that IL-33 plays two opposing actions in mucosal inflammation; the first is a protective action via goblet cell induction, and the second is a pro-inflammatory action as a Th2 cytokine. In the future, it will be necessary to investigate how IL-33 modulates mucosal immune responses in order to develop a novel therapeutic strategy targeting IL-33 and goblet cells for inflammatory bowel disease (23).

\section{References}

1. Schmitz J, Owyang A, Oldham E, et al: IL-33, an interleukin1-like cytokine that signals via the IL-1 receptor-related protein ST2 and induces Thelper type 2-associated cytokines. Immunity 23: 479-490, 2005

2. Arend WP, Palmer G and Gabay C: IL-1, IL-18, and IL-33 families of cytokines. Immunol Rev 223: 20-38, 2008.

3. Sanada S, Hakuno D, Higgins LJ, Schreiter ER, McKenzie AN and Lee RT: IL-33 and ST2 comprise a critical biomechanically induced and cardioprotective signaling system. J Clin Invest 117: 1538-1549, 2007.

4. Nishida A, Andoh A, Imaeda H, et al: Expression of interleukin1-like cytokine interleukin-33 and its receptor complex (ST2L and IL-1RAcP) in human pancreatic myofibroblasts. Gut 59: 531-541, 2009.

5. Marvie P, Lisbonne M, L'Helgoualc'h A, et al: Interleukin-33 overexpression is associated with liver fibrosis in mice and humans. J Cell Mol Med 14: 1726-1739, 2009.

6. Kondo Y, Yoshimoto T, Yasuda K, et al: Administration of IL-33 induces airway hyperresponsiveness and goblet cell hyperplasia in the lungs in the absence of adaptive immune system. Int Immunol 20: 791-800, 2008

7. Zhiguang X, Wei C, Steven R, et al: Over-expression of IL-33 leads to spontaneous pulmonary inflammation in mIL-33 transgenic mice. Immunol Lett 131: 159-165, 2010.
8. Shinoda M, Shin-Ya M, Naito Y, et al: Early-stage blocking of Notch signaling inhibits the depletion of goblet cells in dextran sodium sulfate-induced colitis in mice. J Gastroenterol 45: 608-617, 2010.

9. Kinoshita K, Taupin DR, Itoh H and Podolsky DK: Distinct pathways of cell migration and antiapoptotic response to epithelial injury: structure-function analysis of human intestinal trefoil factor. Mol Cell Biol 20: 4680-4690, 2000.

10. Katoh M: Notch signaling in gastrointestinal tract (Review). Int J Oncol 30: 247-251, 2007.

11. Nakamura T, Tsuchiya $\mathrm{K}$ and Watanabe M: Crosstalk between Wnt and Notch signaling in intestinal epithelial cell fate decision. J Gastroenterol 42: 705-710, 2007.

12. Murayama M, Okamoto R, Tsuchiya K, et al: Musashi-1 suppresses expression of Paneth cell-specific genes in human intestinal epithelial cells. J Gastroenterol 44: 173-182, 2009.

13. Iwasaki M, Tsuchiya K, Okamoto R, et al: Longitudinal cell formation in the entire human small intestine is correlated with the localization of Hath1 and Klf4. J Gastroenterol 46: 191-202, 2011.

14. Yang Q, Bermingham NA, Finegold MJ and Zoghbi HY: Requirement of Math1 for secretory cell lineage commitment in the mouse intestine. Science 294: 2155-2158, 2001.

15. Jensen J, Pedersen EE, Galante P, et al: Control of endodermal endocrine development by Hes-1. Nat Genet 24: 36-44, 2000.

16. Okamoto R, Tsuchiya K, Nemoto Y, et al: Requirement of Notch activation during regeneration of the intestinal epithelia. Am J Physiol Gastrointest Liver Physiol 296: G23-G35, 2009.

17. van Es JH, van Gijn ME, Riccio O, et al: Notch/gamma-secretase inhibition turns proliferative cells in intestinal crypts and adenomas into goblet cells. Nature 435: 959-963, 2005.

18. Mahida YR, Beltinger J, Makh S, et al: Adult human colonic subepithelial myofibroblasts express extracellular matrix proteins and cyclooxygenase-1 and -2. Am J Physiol 273: G1341-G1348, 1997.

19. Andoh A, Fujino S, Bamba S, et al: IL-17 selectively downregulates TNF-alpha-induced RANTES gene expression in human colonic subepithelial myofibroblasts. J Immunol 169: 1683-1687, 2002.

20. Uchiyama K, Naito Y, Takagi T, et al: Carbon monoxide enhance colonic epithelial restitution via FGF15 derived from colonic myofibroblasts. Biochem Biophys Res Commun 391: 1122-1126, 2010.

21. Cooper HS, Murthy SN, Shah RS and Sedergran DJ: Clinicopathologic study of dextran sulfate sodium experimental murine colitis. Lab Invest 69: 238-249, 1993.

22. Rath HC, Herfarth HH, Ikeda JS, et al: Normal luminal bacteria, especially Bacteroides species, mediate chronic colitis, gastritis, and arthritis in HLA-B27/human beta2 microglobulin transgenic rats. J Clin Invest 98: 945-953, 1996.

23. Mayer L: Evolving paradigms in the pathogenesis of IBD. J Gastroenterol 45: 9-16, 2010. 